\title{
Using Optimal Multiple Tuned Liquid Column Dampers for Mitigating the Seismic Response of Structures
}

\author{
Parviz Ahadi, ${ }^{1}$ Mohtasham Mohebbi, ${ }^{2}$ and Kazem Shakeri ${ }^{2}$ \\ ${ }^{1}$ Civil Engineering Department, Islamic Azad University, Germi Branch, Germi, Iran \\ ${ }^{2}$ Engineering Department, University of Mohaghegh Ardabili, Ardabil, Iran
}

Correspondence should be addressed to Parviz Ahadi, pz.ahadi@gmail.com

Received 30 July 2012; Accepted 6 September 2012

Academic Editors: R. S. Jangid and Y. Lai

Copyright (c) 2012 Parviz Ahadi et al. This is an open access article distributed under the Creative Commons Attribution License, which permits unrestricted use, distribution, and reproduction in any medium, provided the original work is properly cited.

\begin{abstract}
Tuned liquid column damper (TLCD) has been used extensively to improve the seismic behavior of structures, and different methods have been proposed for optimal design of TLCDs on linear structures. For improving the effectiveness of TLCDs, multiple tuned liquid column dampers (MTLCDs) have been proposed, and each TLCD has different dynamic parameters. In this paper designing optimal MTLCDs and assessment of its effectiveness in mitigating the response of structures under earthquake excitations have been studied. The parameters of TLCDs have been determined based on minimizing the maximum displacement of structure through solving an optimization problem. Genetic algorithm (GA) has been used for solving the optimization problem. For illustration, the method has been used for designing optimal MTLCDs for a ten-storey linear shear frame subjected to a white noise excitation. According to the results of numerical simulations it could be said that the proposed method for designing optimal MTLCDs has been effective regarding the simplicity and convergence behavior of the method. Based on designing MTLCDs for different values of MTLCDs total mass ratio, it has been concluded that MTLCDs total mass affects significantly the performance of MTMDs where its increasing has led to improve MTLCDs performance. Also, testing the optimal MTLCDs structure under different testing excitations has shown that the performance of MTLCDs depends on the characteristics of earthquakes.
\end{abstract}

\section{Introduction}

During past decades different structural control systems including passive, active, semiactive, and hybrid control mechanisms have been proposed to protect the structures under environmental loads such as wind and earthquake loads [1]. Extensive researches have been conducted theoretically and experimentally to study the efficiency of the proposed control systems whose results show that each of control systems has its individual benefits and limitations regarding the practical and economical issues. In the passive control systems area, it has been found that these kinds of mechanisms are simple and attractive in practical applications. Hence, different mechanisms such as mass damper [2], multiple tuned mass dampers [3-5] viscoelastic dampers [6, 7], tuned liquid dampers, and tuned liquid column dampers [8] have been proposed in this area. The efficiency of single
TLCD in improving the response of structures under wind and earthquake loads has been studied in some researches. Sakai et al. [8] proposed tuned liquid column damper to mitigate excessive vibrations as a passive control system. It is basically a U-tube container with an orifice opening in the middle. The TLCD dissipates the structural vibration energy by the combined action involving the movement of the liquid mass in the container, the restoring force due to gravity on the liquid, and the damping effect due to the orifices as shown in Figure 1. Other researches have studied the performance and design of TLCDs such as optimizing TLCD parameters in controlling structural vibrations including different cross-sectional area in its vertical and horizontal sections $[9,10]$, studying LCVA control performance and effectiveness where the LCVA allows the column crosssection to be nonuniform, research on the effectiveness of the TLCD in reducing the along-wind response of tall 


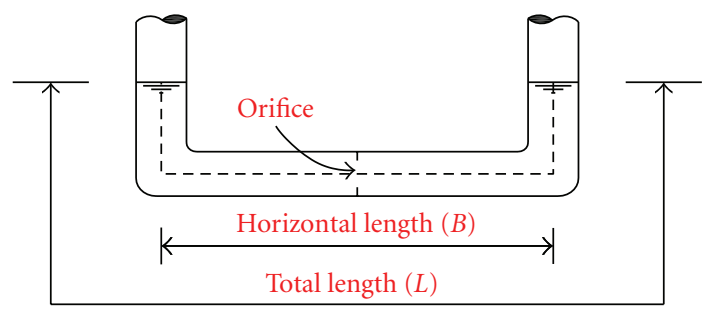

FIGURE 1: Tuned liquid column damper (TLCD) mechanism.

buildings with different mass-stiffness distributions [11, 12], and finding optimum absorber parameters for tuned liquid column damper under wind and earthquake loads [13].

Results of previous researches show that though application of single TLCD could be effective in improving the response of structure under external excitations, it has some limitations such as sensitivity problem to detuning the TLCD frequency, TLCD damping ratio and uncertainty in dynamic properties of main structure and need to heavy mass and large space in installation to tall buildings.

Multiple tuned liquid column dampers (MTLCDs) have been proposed to compensate the limitations of single TLCD [14] where each TLCD has different dynamic characteristics.

In the previous researches, the effectiveness of MTLCDs in reducing the response of linear structures subjected to various external excitations has been studied. Samali et al. [14] studied the performance of MTLCDs in mitigating the vibration of structures under earthquake excitations and concluded that the sensitivity of MTLCDs to uncertainty of structural dynamic parameters is less than a single TLCD. In other researches the effect of different parameters of TLCds such as frequency variation, frequency domain, central frequency, head loss coefficient, and the number of TLCDs on MTLCDs effectiveness has been evaluated $[15,16]$. The results of researches have shown that the TLCDs frequency domain and head loss affect the efficiency of MTLCDs, effectively. Also in the study conducted by Shum and Xu [17] the capability of MTLCDs system in reducing coupled lateral and torsional vibration of structures has been shown. In most of the previous researches on designing MTLCDs the design procedure has some simplifier assumption on TLCDs parameters. In this paper, following the method proposed by Mohebbi et al. [18] for optimal design of multiple tuned mass dampers (MTMDs) on multidegree of freedom (MDOF) structures, an effective method based on optimization procedure has been used for designing optimal MTLCDs.

In the following sections, first the equation of motion of structure MTLCDs will be presented, next to illustrate the procedure of designing optimal MTLCDs, a ten-storey linear shear building frame subjected to white noise excitation, and for different values of TCLDs mass ratio optimal MTLCDs have been designed.

\section{Structure-MTLCDs Equation of Motion}

The equation of motion of a single degree of freedom shear building frame with linear behavior equipped with $N$ tuned

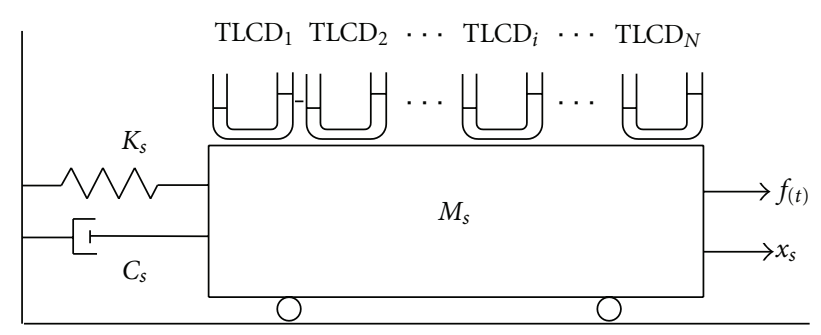

FIGURE 2: Multiple tuned liquid column dampers attached to the structure.

liquid column dampers in parallel configuration located at the top floor, as shown in Figure 2, and subjected to an external force, $f(t)$, can be written in the following form:

$$
\begin{gathered}
\left(\begin{array}{cc}
\bar{M}_{s} & m_{d}^{T} \\
m_{d}^{T} & \underline{\underline{m}}
\end{array}\right)\left[\begin{array}{c}
\ddot{x}_{s} \\
\ddot{x}_{f n}
\end{array}\right]+\left(\begin{array}{cc}
C_{s} & 0 \\
0 & C_{\mathrm{eqn}}
\end{array}\right)\left[\begin{array}{c}
\dot{x}_{s} \\
\dot{x}_{f n}
\end{array}\right]+\left(\begin{array}{cc}
K_{s} & 0 \\
0 & k_{f n}
\end{array}\right)\left[\begin{array}{c}
x_{s} \\
x_{f n}
\end{array}\right] \\
=\left[\begin{array}{c}
f \\
0
\end{array}\right] \\
\bar{M}_{s}=M_{S}+\sum_{i=1}^{N} m_{d n}, \\
m_{d}^{T}=\alpha\left[m_{d 1}, m_{d 2}, \ldots, m_{d N}\right], \\
\underline{m}=\operatorname{diag}\left(\left[m_{d 1}, m_{d 2}, \ldots, m_{d N}\right]\right), \\
C_{s}=2 M_{s} \zeta_{s} \omega_{s}, \\
\underline{C}_{\mathrm{eqi}}=\operatorname{diag}\left(\left[c_{d 1}, c_{d 2}, \ldots, c_{d N}\right]\right), \\
\underline{K}_{f i}=\operatorname{diag}\left(\left[k_{d 1}, k_{d 2}, \ldots, k_{d N}\right]\right), \\
m_{d i}=\rho A_{f i} L_{f i}, \\
c_{d i}=2 m_{d i} \zeta_{f i} \omega_{d i}, \\
k_{d i}=2 \rho A_{f i} g
\end{gathered}
$$

where $M_{s}, C_{s}$, and $K_{s}$ are structure mass, damping and stiffness, respectively. $x_{s}, \dot{x}_{s}$, and $\ddot{x}_{s}$ are structure displacement, velocity, and acceleration vectors, respectively. Also $\zeta_{f i}$ and $\omega_{d i}$ are the damping ratio and natural frequency of the $i$ th TLCD. $\rho=$ liquid density, $L_{f i}$ and $A_{f i}$ are total length of liquid in the container and cross-sectional area of tube. Also $\alpha=B_{f i} / L_{f i}$ where $B_{f i}$ is the horizontal portion of liquid in the $i$ th TLCD container, respectively.

\section{Designing Optimal MTLCDS}

In this paper following the method proposed by Mohebbi et al. [18] for optimal design of multiple tuned mass dampers (MTMDs) for MDOF structures subjected to earthquake excitation, an effective method has been proposed for optimal design of MTLCDs on multidegree-of-freedom linear structures subjected to any desired excitation. In this method, for designing MTLCDs system, an optimization 
problem has been defined where the minimization of the maximum structure displacement $\left(x_{\max }\right)$ is considered as objective and the parameters of TLCDs such as mass, total length, and head loss as variables.

The optimization problem in the case of using MTLCDs has a large number of variables as well as is sophisticated; hence using traditional optimization techniques such as gradient-based methods is considerably complicated. In this paper it has been decided to use a powerful algorithm to solve the problem where genetic algorithm (GA) has been utilized for solving the optimization problem.

\section{Genetic Algorithms (GAs)}

To solve the optimization problem iterations between optimizer that decides the next searching points and analyzer that determines the value of object function should be performed. In the traditional optimization method the domain is searched using the gradient of the objective function and the limitation of this method arises when the functions of objective function and the constraints of the optimization problem are not continuous, and it is not possible to calculate the gradient of the functions.

Genetic algorithm (GA) which has been developed by Holland [19] is a computational method and has proven to be suitable and successful direct searching algorithm for solving linear and/or nonlinear optimization problems where the derivatives of the objective function and/or constraints of the problem are not available or are hard to compute.

There are three genetic algorithm operators including selection, crossover and mutation. In every generation, a set of chromosomes is selected for mating based on their relative fitness. The fitters are given more chance of passing their genes into the next generation. This process of natural selection is operated by selection. The selected individuals are then chosen randomly through crossover to produce offspring, Crossover produces new individuals that have some parts of both parents genetic material.

The role of mutation operator is to help the GA to escape from local minima and to provide a guarantee that the probability of searching any given string will never be zero.

4.1. Real-Valued Coding. In GAs chromosomes evolving under a certain environment are represented by bit strings or real-valued coding. In the early stages of string coding the variables were represented in binary format $[20,21]$. Whilst binary-coded GAs appear to be more suitable when solving complex problems, they have some drawbacks in taking continuous problems, and it has been shown that for realvalued numerical optimization problems, real-valued coding representations offer certain advantages, such as simpler programming, less memory required, no need to convert chromosomes, and greater freedom to use different genetic operators, over binary versions $[22,23]$.

4.2. The Objective and Fitness Function. The objective function is used to provide a measure of how individuals have performed in the problem domain. In the case of a minimization problem, the fit individuals will have the lowest numerical value of the associated objective function.

One of the biggest problems for GAs is handling constraints of optimization problems. There are several studies of handling constrains in GAs. The simple method of handling constraints is a penalty term that is added to the objective function for the degree of violation of constraints. In the penalty method the objective function which has to be minimized is obtained by combining the objective function $f$ and the constraints functions $g_{i}$ for $n$ number of constraints, given by

$$
F=f+\sum_{c=1}^{n} p c_{i} \max \left[0, g_{i}\right]
$$

where $f=$ objective function, $p c_{i}=i$ th penalty coefficient, and $g_{i}=i$ th constraint violation.

4.3. Selection. Selection is the process of determining the number of times or trials; a particular individual is chosen for reproduction and, thus, the number of offspring that an individual will produce. The selection procedure used by GA is based on the fitness of each individual. Different methods have been proposed for selection operator in binary or real value coding representations such as roulette wheel, rank and stochastic universal sampling (SUS) methods [24].

In this paper stochastic universal sampling method [24] is used for selection of the individuals for reproduction according to their fitness in the current population.

4.4. Crossover (Recombination). The basic operator for producing new individuals in the GA is that of crossover. Like its counterpart in nature, crossover produces new individuals that have some parts of both parents' genetic material. For binary-coded GA, the crossover is carried out by swapping the individual contents at the crossover site. Details and several methods of this operation (such as one-point, multiplepoint, and uniform crossover) can be fined in references also for real-coded GA; several types of recombination such as intermediate, line, and uniform recombination have been proposed [21]. In this study intermediate recombination method [25] has been used for crossover.

4.5. Mutation. The role of mutation is often seen as providing a guarantee that the probability of searching any given string will never be zero and acting as a safety net to recover good genetic materials that may be lost through the action of selection and crossover [20]. Many variations on the mutation have been proposed, including random, boundary, nonuniform mutation [21].

4.6. Reinsertion. To maintain the size of the original population, the new chromosomes have to be reinserted into the old population. An insertion rate, $\eta$, determines the number of newly produced chromosomes inserted in the old population. Also in this paper the elitist strategy has been used which allows some of the best chromosomes in 


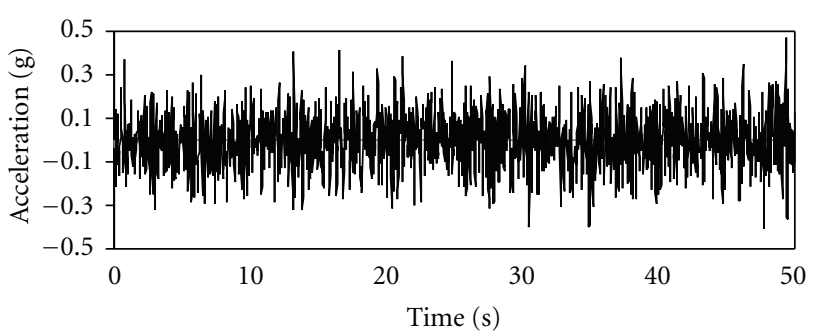

FIgure 3: White noise excitation, $W(t)$, with PGA $=0.475 \mathrm{~g}$.

the current population to go to the next generation without modification.

\section{Numerical Example}

In this paper, to assess the effectiveness of the proposed method in designing optimal MTLCDs as well as to evaluate the effect of different parameters of MTLCDs on its performance, a ten-storey shear frame with uniform properties for all stories has been modelled assuming linear material behavior for structure and TLCDs. The properties of each storey are as follows: $m_{i}=360$ tons, $k_{i}=650 \mathrm{MN} / \mathrm{m}$, and $c_{i}=6.2 \mathrm{MN} \cdot \mathrm{s} / \mathrm{m}$. The height of all stories has been considered $3 \mathrm{~m}$, and the section parameters of columns are determined so that the assumed stiffness has been achieved.

By assuming a specified value for the total mass ratio, $\mu$, uniform distribution for TLCDs mass has been considered.

The structure has been subjected under a filtered white noise excitation, $W(t)$, shown in Figure 3 by considering MTLCDs in parallel configuration located at the top of structure. To illustrate the proposed method for designing the optimal MTLCDs, five TLCDs with uniform mass distribution have been considered where the total mass ratio is $\mu=2 \%$. For solving the optimization problem, the following parameters have been considered for GA: number of individuals in each generation $=25$, number of elites in each generation $=2$, number of the newborns in each generation $=25$, and mutation rate $=0.04$.

The optimization problem has been solved using GA and to guarantee the accuracy of optimization procedure, different runs have been done in GA. Figure 4 shows the variation of the best fitness during 500 generations of GA for two runs. According to the result it can be said that different runs have ended with the same optimum answer but with different convergence speed. This result shows the convergence and simplicity behavior of the proposed method in designing optimal MTLCDs.

The uncontrolled structure and controlled structure with optimal MTLCDs have been subjected to white noise excitation, and the maximum displacements of controlled and uncontrolled structures have been shown in Figure 5 for different storeys. Based on the results shown in Figure 5, it have been found that by using MLCDs when $\mu=2 \%$ and $N=5$, it is possible to have about $50 \%$ reduction in maximum displacement.

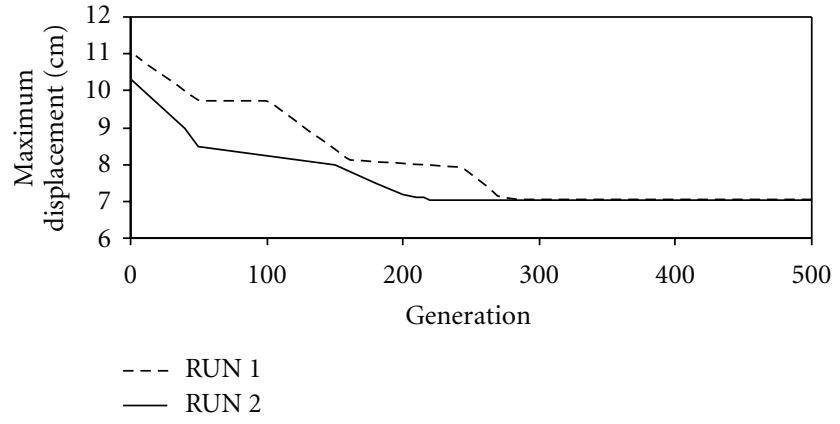

FIGURE 4: Convergence of GA to optimum answer for different runs.

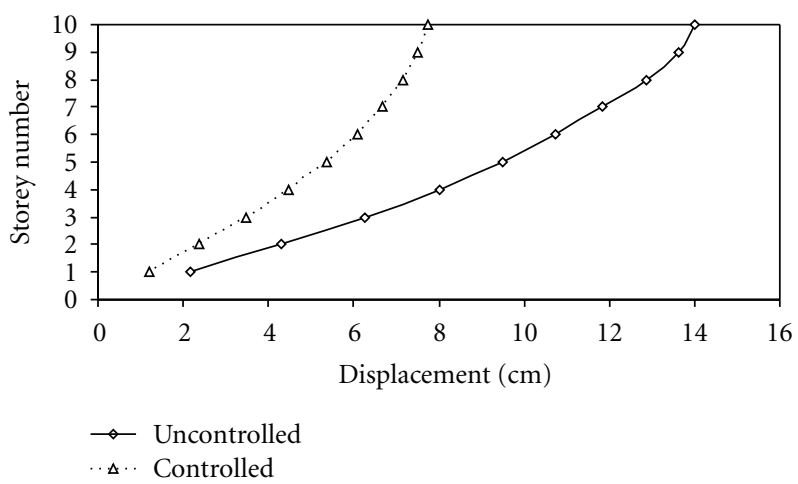

FIGURE 5: Normalized response of controlled structure under $W(t)$ excitation when $\mu=2 \%$ and $N=5$.

To assess the effectiveness of optimal MTLCDs designed using $W(t)$ as design excitation in mitigating the response of structure under other real earthquakes which are different in peak ground acceleration, and frequency content with design excitation, the uncontrolled and controlled structure have been tested under El-Centro (1940, PGA = 0.34 g) and Hachinohe (1968; PGA $=0.23 \mathrm{~g}$ ) as Far-Field as well as Northridge (1994; PGA $=0.84 \mathrm{~g})$ and Kobe $(1995,0.83 \mathrm{~g})$ as Near-Field earthquakes. The maximum displacement of controlled structure has been shown in Figure 6 for different storeys under testing earthquakes.

Results show that using MTLCDs has led not only to decrease the maximum values of structure displacement but also the maximum displacement of all stories has been decreased. Also it has been found that the effectiveness of MTLCDs depends on the characteristics of earthquake, where in this case study, the best performance has been achieved under El-Centro (1940) excitation as a Far-Field record. Based on the results it could be said that in designing MLCDs for a special area, the design earthquake of that area should be used as design record.

5.1. Designing Optimal TLCDs for Different Mass Ratio. Following the same procedure explained for $\mu=2 \%$ and $N=5$, for other values of MTLCDs mass ratio assuming uniform mass distribution for TLCDs, optimal MTLCDs has been designed when the structure subjected to $W(t)$. The maximum displacement, drift, acceleration and 


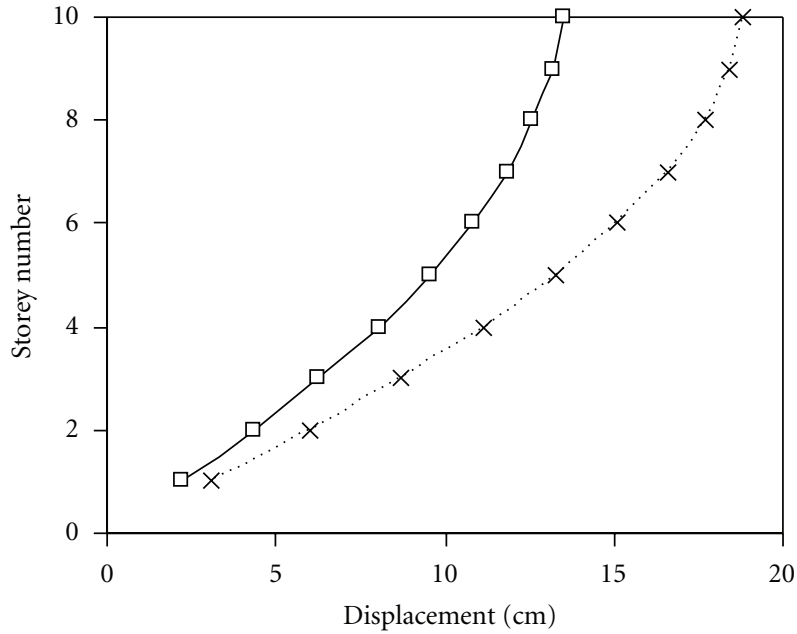

(a)

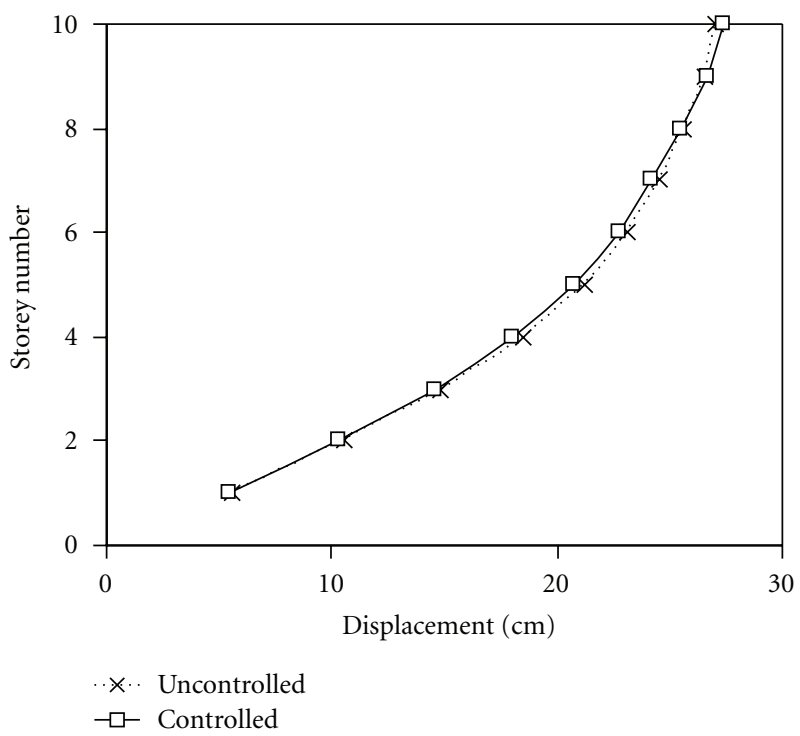

(c)

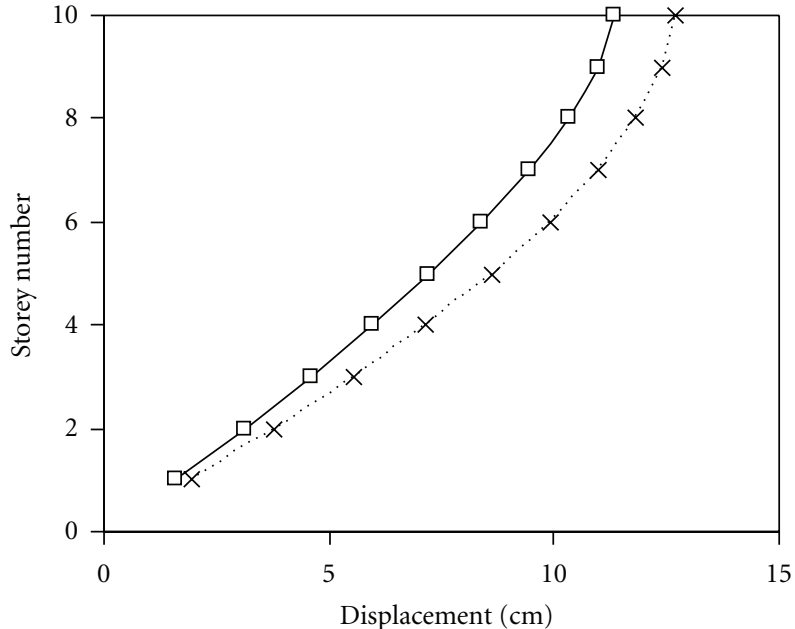

(b)

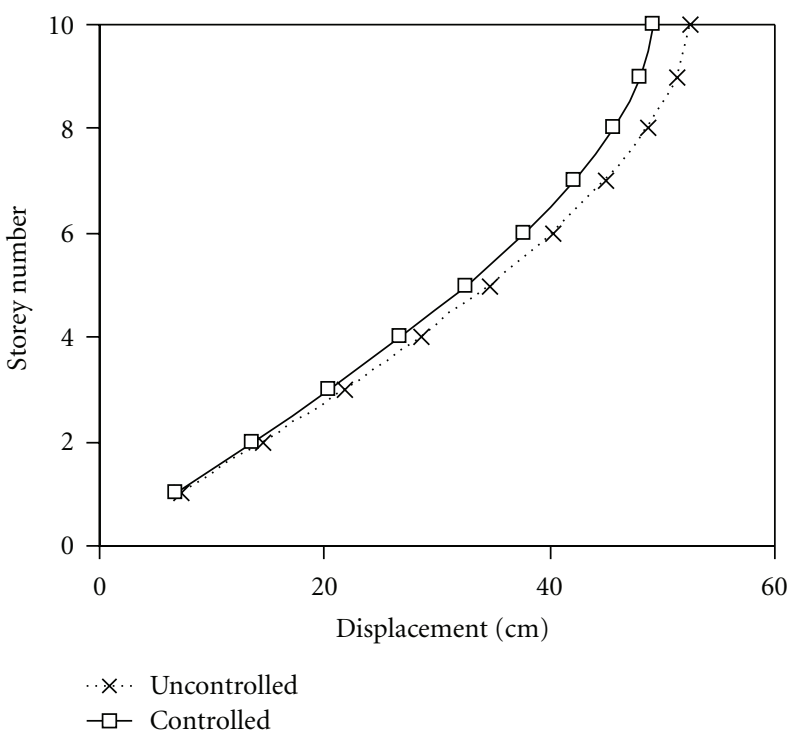

(d)

Figure 6: Maximum displacement of controlled structure under (a) El-Centro (1940), (b) Hachinohe (1968), (c) Northridge (1994) and (d) Kobe (1995) testing excitations when $\mu=2 \%$ and $N=5$.

displacement root mean square (RMS) of controlled frame has been divided to maximum uncontrolled response and shown in Figure 7 for different values of total mass ratio, $\mu$. Results show that increasing the mass ratio has led to improving the performance of MTLCDs in reducing the maximum response of structure. Also it has been found that though minimizing the maximum displacement has been considered as objective in designing optimal MTLCDs, the maximum acceleration has been reduced, too.

\section{Conclusions}

In this paper optimal design of multiple tuned liquid column dampers (MTLCDs) and its effectiveness in mitigating the response of structures subjected to earthquake excitation have been studied. Designing MTLCDs has been based on minimizing the maximum displacement of structure where the parameters of MTLCDs have been determined through solving an optimization problem. Genetic algorithm (GA) has been used for solving the optimization problem to determine the parameters of MTLCDs. To assess the effectiveness of the method, a ten-storey linear shear frame subjected to a white noise excitation and optimal MTMDs has been designed for different values of MTLCDs total mass ratio values. According to the results of numerical simulations, the capability of the MTLCDs design method has been shown regarding the simplicity and convergence behavior of the method. Also it has been found that genetic algorithm (GA) has been a powerful tool in solving complex optimization problem of MTLCDs design with a large number of variables. Designing optimal MTLCDs for 


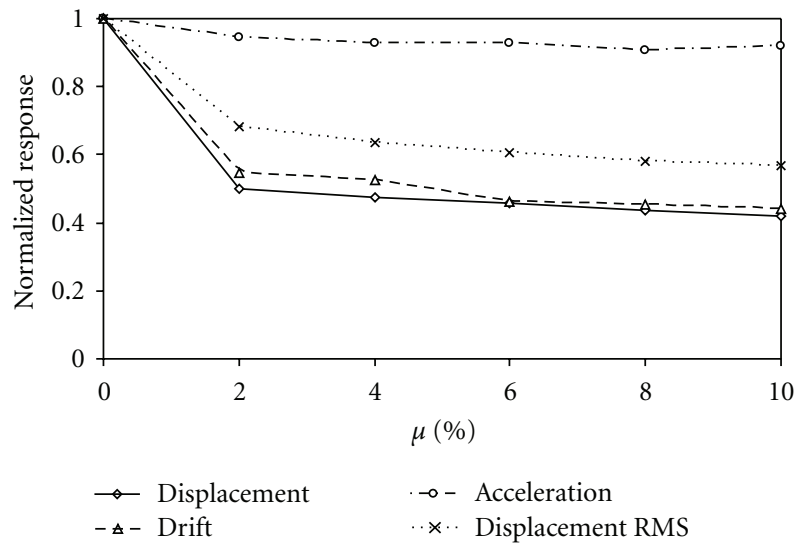

FIGURE 7: Normalized maximum response of controlled structure under $W(t)$ for different values of total mass ratio.

different mass ratios shows that MTLCDs could be effective in reducing the maximum response of the structure where its performance is affected by total mass ratio of MTLCDs. It has been found that increasing the total mass of MTLCDs has improved the performance of MTMDs in reducing the response of structure. Optimal MTLCDs have been tested under both Far-Field and Near-Field earthquakes which are different in frequency content and peak ground acceleration (PGA) with design excitation where the results show that MTLCDs efficiency under testing records depends on the characteristics of earthquakes.

\section{References}

[1] B. F. Spencer and S. Nagarajaiah, "State of the art of structural control," Journal of Structural Engineering, vol. 129, no. 7, pp. 845-856, 2003.

[2] T. Soong and G. F. Dargush, Passive Energy Dissipation Systems in Structural Engineering, John Wiley \& Sons, Chichester, UK, 1997.

[3] S. V. Bakre and R. S. Jangid, "Optimum parameters of tuned mass damper for damped main system," Structural Control and Health Monitoring, vol. 14, no. 3, pp. 448-470, 2007.

[4] V. B. Patil and R. S. Jangid, "Optimum multiple tuned mass dampers for the wind excited benchmark building," Journal of Civil Engineering and Management, vol. 17, pp. 540-557, 2011.

[5] T. P. Bandivadekar and R. S. Jangid, "Mass distribution of multiple tuned mass dampers for vibration control of structures," International Journal of Civil and Structural Engineering, vol. 3, pp. 70-84, 2012.

[6] Z. D. Xu, H. T. Zhao, and A. Q. Li, "Optimal analysis and experimental study on structures with viscoelastic dampers," Journal of Sound and Vibration, vol. 273, no. 3, pp. 607-618, 2004.

[7] Z. D. Xu, "Earthquake mitigation study on viscoelastic dampers for reinforced concrete structures," JVC/Journal of Vibration and Control, vol. 13, no. 1, pp. 29-43, 2007.

[8] F. Sakai, S. Takaeda, and T. Tamaki, "Tuned liquid column damper-new type device for suppression of building vibration," in Proceedings of the International Conference on HighRise Buildings, pp. 926-931, Nanjing, China, 1989.
[9] H. Gao, K. C. S. Kwok, and B. Samali, "Optimization of tuned liquid column dampers," Engineering Structures, vol. 19, no. 6, pp. 476-486, 1997.

[10] C. C. Chang and C. T. Hsu, "Control performance of liquid column vibration absorbers," Engineering Structures, vol. 20, no. 7, pp. 580-586, 1998.

[11] T. Balendra, C. M. Wang, and G. Rakesh, "Vibration control of various types of buildings using TLCD," Journal of Wind Engineering and Industrial Aerodynamics, vol. 83, pp. 197-208, 1999.

[12] T. Balendra, C. M. Wang, and G. Rakesh, "Effectiveness of TLCD on various structural systems," Engineering Structures, vol. 21, no. 4, pp. 291-305, 1999.

[13] K. Y. Swaroop and A. Kareem, "Optimum absorber parameters for tuned liquid column dampers," Journal of Structural Engineering, vol. 126, no. 8, pp. 906-915, 2000.

[14] B. Samali, D. Templeton, and K. C. S. Kwok, "The effectiveness of multiple tuned liquid column dampers in controlling vibration of tall buildings subject to earthquake excitation," in Proceedings of the 2nd International Conference on Motion and Vibration Control,, pp. 120-125, Yokohama, Japan, 1994.

[15] P. A. Hitchcock, K. C. S. Kwok, R. D. Watkins, and B. Samali, "The effectiveness of a multiple liquid column vibration absorber," in Proceedings of the 4th Workshop on Wind Engineering, Australian Wind Engineering Society, pp. 81-86, Sydney, Australia, 1994.

[16] H. Gao, K. S. C. Kwok, and B. Samali, "Characteristics of multiple tuned liquid column dampers in suppressing structural vibration," Engineering Structures, vol. 21, no. 4, pp. 316-331, 1999.

[17] K. M. Shum and Y. L. Xu, "Multiple tuned liquid column dampers for reducing coupled lateral and torsional vibration of structures," Engineering Structures, vol. 26, no. 6, pp. 745$758,2004$.

[18] M. Mohebbi, K. Shakeri, Y. Ghanbarpour, and H. Majzub, "Designing optimal multiple tuned massdampers using Genetic Algorithms (GAs) for mitigating the seismic response of structures," Journal of Vibration and Control. In press.

[19] J. H. Holland, Adaptation in Natural and Artificial Systems, The University of Michigan Press, Ann Arbor, Mich, USA, 1975.

[20] D. E. Goldberg, Genetic Algorithms in Search, Optimization and Machine Learning, Addison-Wesley, Reading, Mass, USA, 1989.

[21] Z. Michalewicz, Genetic Algorithms + Data Structures = Evolution Programs, Springer, New York, NY, USA, 1996.

[22] W. M. Jenkins, "A decimal-coded evolutionary algorithm for constrained optimization," Computers and Structures, vol. 80, no. 5-6, pp. 471-480, 2002.

[23] Y. Arfiadi and M. N. S. Hadi, "Optimal direct (static) output feedback controller using real coded genetic algorithms," Computers and Structures, vol. 79, no. 17, pp. 1625-1634, 2001.

[24] J. E. Baker, "Reducing bias and inefficiency in the selection algorithm," in Proceedings of the 2nd International Conference on Genetic Algorithm (ICGA '87), vol. 2, pp. 14-21, Cambridge, Mass, USA, July 1987.

[25] H. Mühlenbein and D. Schlierkamp-Voosen, "Predictive models for the breeder genetic algorithm: I. Continuous parameter optimization," Evolutionary Computation, vol. 1, no. 1, pp. 2549, 1993. 

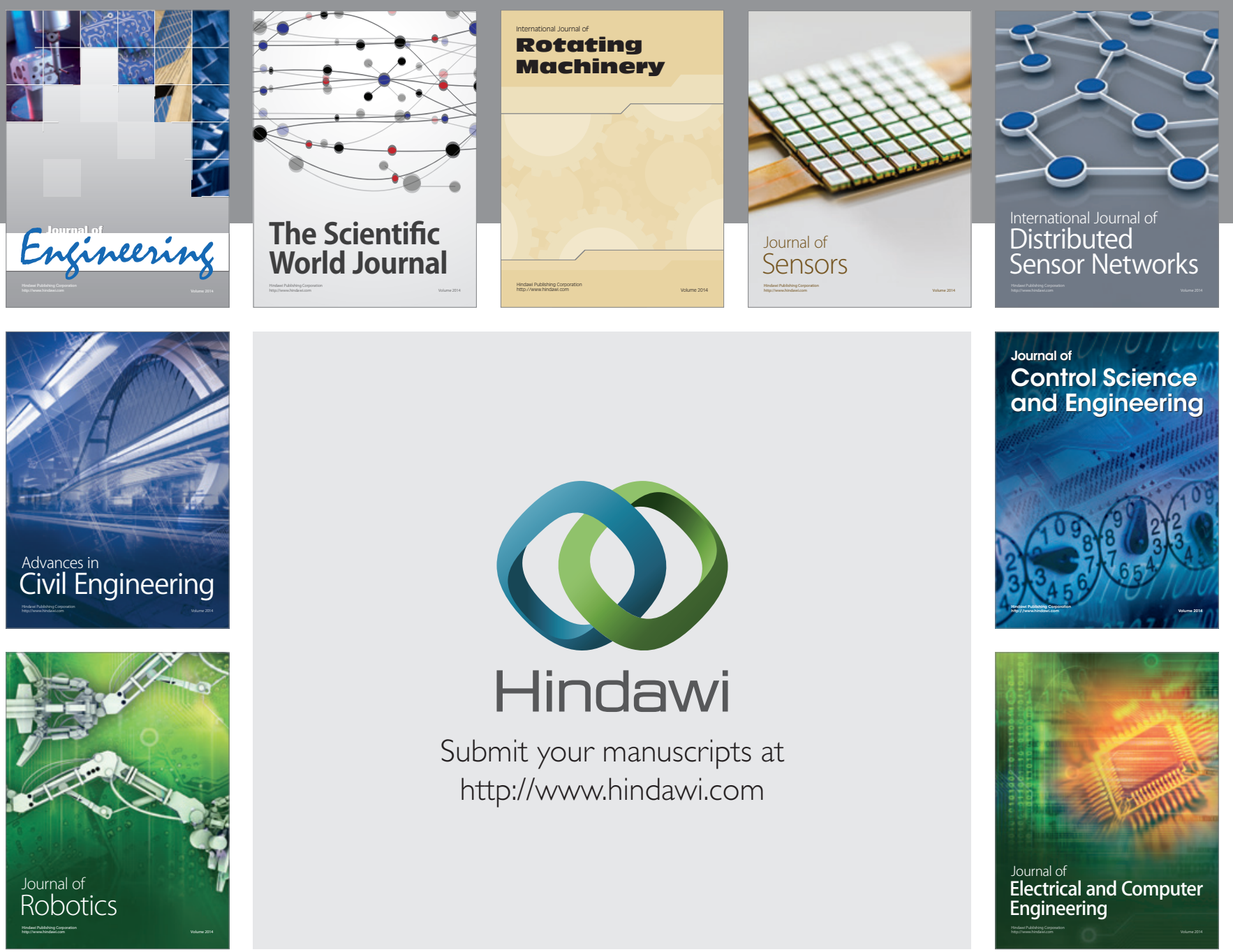

Submit your manuscripts at

http://www.hindawi.com
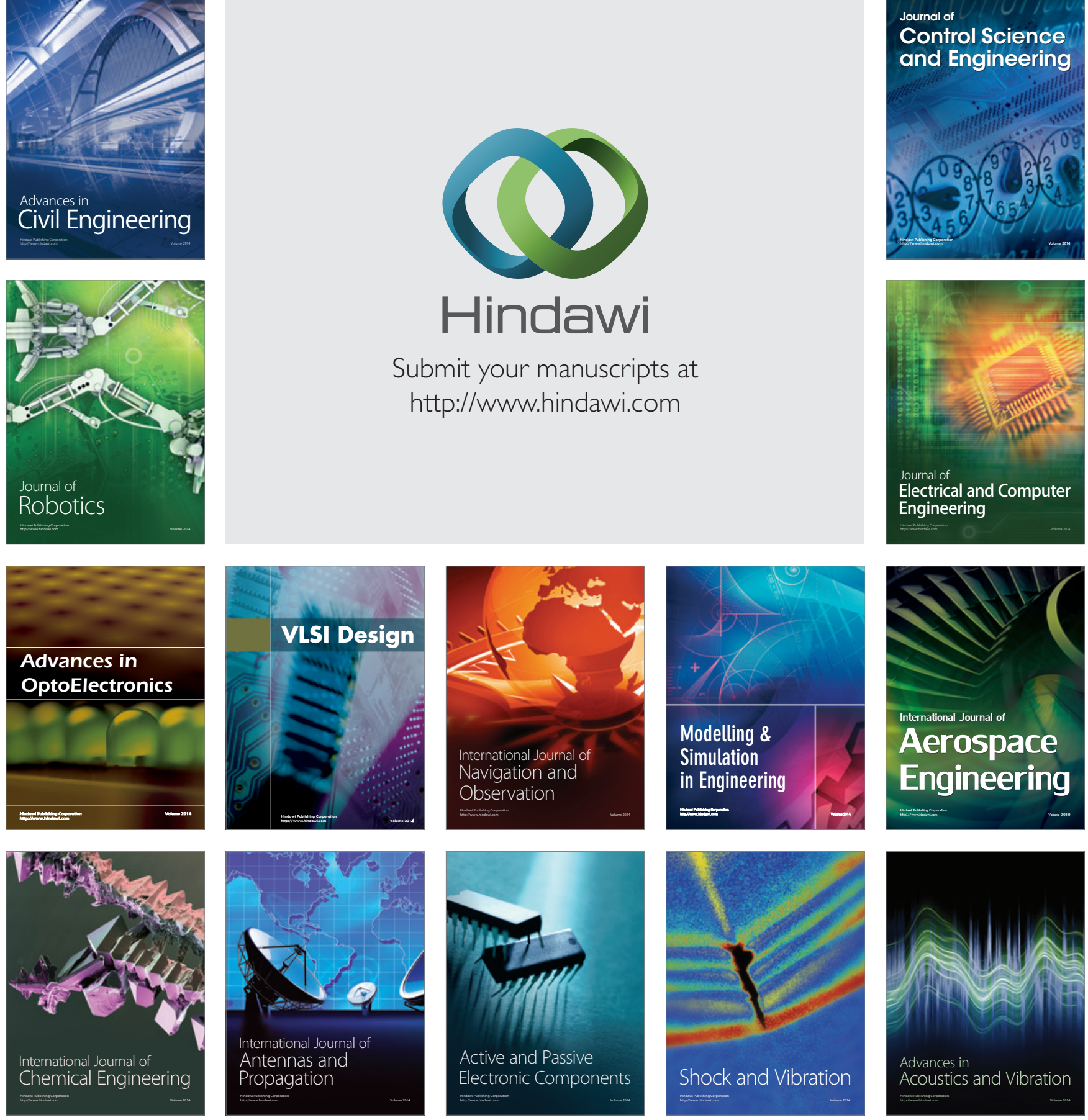